\title{
The molecular envelope of the red supergiant VY CMa
}

\author{
S. Muller ${ }^{1}$, Dinh-Van-Trung ${ }^{1}$, C. Muthumariappan ${ }^{1}$, J. Lim ${ }^{1}$, \\ N. Hirano ${ }^{1} \&$ S. Kwok ${ }^{2,1}$ \\ ${ }^{1}$ Academia Sinica - Institute of Astronomy \& Astrophysics, Taiwan \\ ${ }^{2}$ Department of Physics, Faculty of Science, University of Hong Kong
}

\begin{abstract}
Millimeter and submillimeter interferometry allows to probe the environment around evolved massive stars, where optical and NIR studies might be severely limited by large extinctions due to dusty environments. We present here the morphology and kinematics of the molecular envelope around the red supergiant star VY CMa. We have used the Submillimeter Array (SMA) to map the ${ }^{12} \mathrm{CO}(\mathrm{J}=2-1)$ line and $1.3 \mathrm{~mm}$ continuum emissions with an unprecedent resolution of $<2$ " or $3000 \mathrm{AU}$. While the line emission presents a roughly circular shape with a radius of $\sim 6000 \mathrm{AU}$, the velocity structure is markedly different from that of a simple spherically expanding shell. In particular, there is a significant velocity gradient in the East-West direction, confirming the presence of a bipolar outflow.
\end{abstract}

Keywords. supergiants - circumstellar matter

The last phase in the evolution of massive stars $\left(>15 \mathrm{M}_{\odot}\right)$ is characterized by a very short timescale and drastic changes in their immediate circumstellar environment. Periods of large mass loss form a thick circumstellar envelope of molecular gas and dust, heavily obscuring the inner regions of the nebula.

VY CMa is one the closest red supergiant $(\mathrm{D}=1.5 \mathrm{kpc})$. The star is very luminous $\left(\sim 10^{5} \mathrm{~L}_{\odot}\right)$, massive $\left(\geqslant 15 \mathrm{M}_{\odot}\right)$, and presents a huge mass loss rate $\left(\sim 10^{-4} \mathrm{M}_{\odot} / \mathrm{yr}\right)$. The optical/infrared morphology of the nebulosity around VY CMa is very complex, showing bright knots, arcs and filaments few arcsec across in a very complicated arrangement (Monnier et al. 1999, Smith et al. 2001, Humphreys et al. 2005) and suggesting episodic mass loss events. These images however rely on scattered light and may not reflect the real geometry of the inner envelope. To investigate the structure, kinematics, physical properties, chemistry and evolution of the circumstellar envelope, one can turn to the thermal molecular emission, using the $\mathrm{mm} / \mathrm{submm}$ interferometers as unique observing tools.

In this contribution, we report high angular resolution observations of the circumstellar molecular envelope of VY CMa obtained with the SubMillimeter Array $\dagger$. We have mapped the emission of the ${ }^{12} \mathrm{CO}(\mathrm{J}=2-1)$ molecular line, as well as the $1.3 \mathrm{~mm}$ continuum emission. Observations were obtained in July 2004 and January 2005 in the compact and extended configuration respectively. The final synthesized beam results in an angular resolution higher than 2 ", ie. $<3000$ AU at the distance of VY CMa.

We present in Fig. 1a the integrated intensity of the ${ }^{12} \mathrm{CO}(\mathrm{J}=2-1)$ emission. It reveals a rather circular shape, with an extent to roughly 6000 AU from the central star. The kinematics of the molecular gas is however not consistent with a spherically expanding shell. A clear velocity gradient is found on the East-West direction as illustrated by the

$\dagger$ The Submillimeter Array is a joint project between the Smithsonian Astrophysical Observatory and the Academia Sinica Institute of Astronomy and Astrophysics and is funded by the Smithsonian Institution and the Academia Sinica. 
position - velocity diagram on Fig.1b. We interpret this geometry by a central shell + EW outflows as already suggested by Shinnaga et al. 2003 based on $\mathrm{SiO}(\mathrm{J}=1-0)$ polarimetric spectral-line imaging.

We are currently working on modelling to constrain the physical properties of the molecular gas in the central low velocity component and in the high velocity outflows. Our preliminary results, taking into account the $\mathrm{CO}$ spectra with transitions up to $\mathrm{CO}(\mathrm{J}=6-$ 5) observed by Kemper et al. (2003), argue in favor of a low velocity $\left(\mathrm{V}_{\exp }=15 \mathrm{kms}^{-1}\right)$ central component, with a mass of $0.04 \mathrm{M}_{\odot}$ (adopting a $\mathrm{CO}$ abundance of $310^{-4}$ as compared to $\mathrm{H}_{2}$ ), an extension radius of $10^{17} \mathrm{~cm}$ and an average mass loss rate of 2 $10^{-5} \mathrm{M}_{\odot} / \mathrm{yr}$ over a timescale of $\sim 2000 \mathrm{yr}$. Concerning the high velocity $\left(\mathrm{V}_{\exp }=45\right.$ $\mathrm{kms}^{-1}$ ) component, we estimate its mass to be twice larger, leading to a total mass of the molecular envelope of $\sim 0.12 \mathrm{M}_{\odot}$.

On the other hand, the $1.3 \mathrm{~mm}$ continuum emission is unresolved by our synthesized beam and accounts for a dust mass of $\sim 1.410^{-3} \mathrm{M}_{\odot}$. We estimate a corresponding lower limit for the mass loss rate of $410^{-4} \mathrm{M}_{\odot} / \mathrm{yr}$, with a time scale of $\sim 300 \mathrm{yr}$, supporting the idea of episodic and high mass loss events.

a)

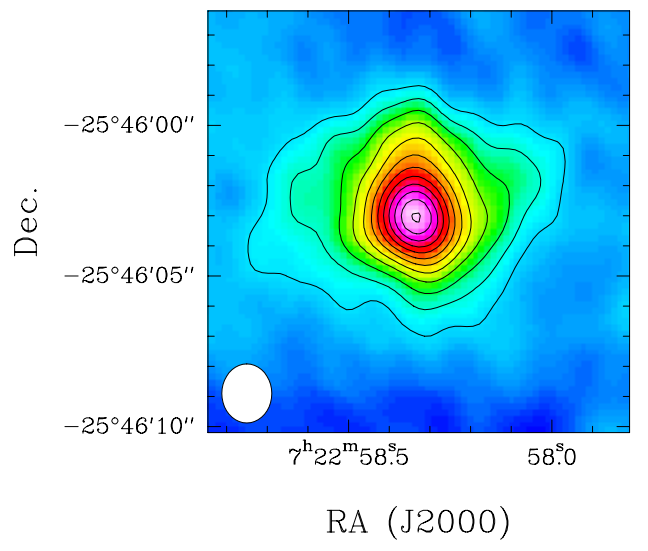

b)

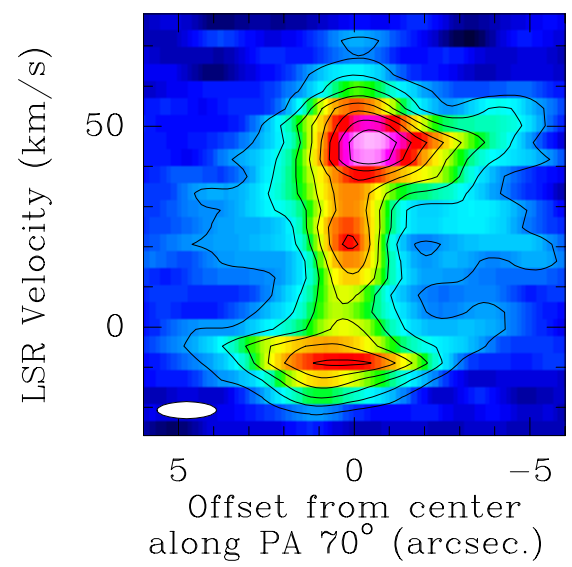

Figure 1. a) Integrated intensity of the ${ }^{12} \mathrm{CO}(\mathrm{J}=2-1)$ emission of the circumstellar envelope of VY CMa. Contours levels every $150 \mathrm{~K} . \mathrm{km} / \mathrm{s}(10 \sigma)$. The size of the synthesized beam is indicated by the ellipse in the bottom left corner. $b$ ) Position - velocity diagram along the outflows, taken at a direction of $70^{\circ}$ from North, anticlockwise.

\section{References}

Humphreys, R. M., Davidson, K. \& Ruch, G. 2005 AJ 129, 492

Kemper, F., Stark, R., Justtanont, K. et al. 2003, A\&A 407, 609

Monnier, J.D., Tuthill, P.G., Lopez, B. et al. 1999 ApJ 512, 351

Shinnaga, H., Claussen, M.J., Lim, J. et al. 2003 in ASSL Vol. 283, Mass-Losing Pulsating Stars and their Circumstellar Matter, Eds. Y. Nakada, M. Honma \& M. Seki (Dordrecht: Kluwer), 393

Smith, N., Humphreys, R.M., Davidson, K. et al. 2001 AJ 121, 1111 